\title{
Time-Scale-Dependent Criteria for the Existence of Positive Solutions to $p$-Laplacian Multipoint Boundary Value Problem
}

\author{
Wenyong Zhong ${ }^{1}$ and Wei Lin ${ }^{2}$ \\ ${ }^{1}$ School of Mathematics and Computer Sciences, Jishou University, Hunan 416000, China \\ 2 Shanghai Key Laboratory of Contemporary Applied Mathematics, School of Mathematical Sciences, \\ Fudan University, Shanghai 200433, China
}

Correspondence should be addressed to Wei Lin, wlin@fudan.edu.cn

Received 1 May 2010; Revised 23 July 2010; Accepted 30 July 2010

Academic Editor: Alberto Cabada

Copyright (C) $2010 \mathrm{~W}$. Zhong and W. Lin. This is an open access article distributed under the Creative Commons Attribution License, which permits unrestricted use, distribution, and reproduction in any medium, provided the original work is properly cited.

\begin{abstract}
By virtue of the Avery-Henderson fixed point theorem and the five functionals fixed point theorem, we analytically establish several sufficient criteria for the existence of at least two or three positive solutions in the $p$-Laplacian dynamic equations on time scales with a particular kind of $p$-Laplacian and $m$-point boundary value condition. It is this kind of boundary value condition that leads the established criteria to be dependent on the time scales. Also we provide a representative and nontrivial example to illustrate a possible application of the analytical results established. We believe that the established analytical results and the example together guarantee the reliability of numerical computation of those $p$-Laplacian and $m$-point boundary value problems on time scales.
\end{abstract}

\section{Introduction}

The investigation of dynamic equations on time scales, originally attributed to Stefan Hilger's seminal work $[1,2]$ two decades ago, is now undergoing a rapid development. It not only unifies the existing results and principles for both differential equations and difference equations with constant time stepsize but also invites novel and nontrivial discussions and theories for hybrid equations on various types of time scales [3-11]. On the other hand, along with the significant development of the theories, practical applications of dynamic equations on time scales in mathematical modeling of those real processes and phenomena, such as the population dynamics, the economic evolutions, the chemical kinetics, and the neural signal processing, have been becoming richer and richer $[12,13]$. 
As one of the focal topics in the research of dynamic equations on time scales, the study of boundary value problems for some specific dynamic equations on time scales recently has elicited a great deal of attention from mathematical community [14-33]. In particular, a series of works have been presented to discuss the existence of positive solutions in the boundary value problems for the second-order equations on time scales [14-21]. More recently, some analytical criteria have been established for the existence of positive solutions in some specific boundary value problems for the $p$-Laplacian dynamic equations on time scales [22,33].

Concretely, He [25] investigated the following dynamic equation:

$$
\left(\phi_{p}\left(u^{\Delta}(t)\right)\right)^{\nabla}+h(t) f(u)=0, \quad t \in[0, T]_{\mathbb{T}}
$$

with the boundary value conditions

$$
u^{\Delta}(0)=0, \quad u(T)+B_{0}\left(u^{\Delta}(\eta)\right)=0 .
$$

Here and throughout, $\mathbb{T}$ is supposed to be a time scale; that is, $\mathbb{T}$ is any nonempty closed subset of real numbers in $\mathbb{R}$ with order and topological structure defined in a canonical way. The closed interval in $\mathbb{T}$ is defined as $[a, b]_{\mathbb{T}}=[a, b] \cap \mathbb{T}$. Accordingly, the open interval and the half-open interval could be defined, respectively. In addition, it is assumed that $0, T \in \mathbb{T}$, $\eta \in(0, \rho(T))_{\mathbb{T}}, f \in C_{\mathrm{ld}}([0, \infty),[0, \infty)), h \in C_{\mathrm{ld}}\left((0, T)_{\mathbb{T}},(0, \infty)\right)$, and $\underline{b} x \leqslant B_{0}(x) \leqslant \bar{b} x$ for some positive constants $\underline{b}$ and $\bar{b}$. Moreover, $\phi_{p}(u)$ is supposed to be the $p$-Laplacian operator, that is, $\phi_{p}(u)=|u|^{p-2} u$ and $\left(\phi_{p}\right)^{-1}=\phi_{q}$, in which $p>1$ and $1 / p+1 / q=1$. With these configurations and with the aid of the Avery-Henderson fixed point theorem [34], He established the criteria for the existence of at least two positive solutions in (1.1) fulfilling the boundary value conditions (1.2).

Later on, $\mathrm{Su}$ and $\mathrm{Li}$ [24] discussed the dynamic equation (1.1) which satisfies the boundary value conditions

$$
u^{\Delta}(0)=0, \quad u(T)+B_{0}\left(\sum_{i=1}^{m-2} b_{i} u^{\Delta}\left(\xi_{i}\right)\right)=0
$$

where $\xi \in(0, T), 0<\xi_{1}<\xi_{2}<\cdots<\xi_{m-2}<T$, and $b_{i} \in[0, \infty)$ for $i=1,2, \ldots,(m-2)$. By virtue of the five functionals fixed point theorem [35], they proved that the dynamic equation (1.1) with conditions (1.3) has three positive solutions at least. Meanwhile, He and Li in [26], studied the dynamic equation (1.1) satisfying either the boundary value conditions

$$
u(0)-B_{0}\left(u^{\Delta}(0)\right)=0, \quad u^{\Delta}(T)=0,
$$

or the conditions

$$
u^{\Delta}(0)=0, \quad u(T)+B_{0}\left(u^{\Delta}(T)\right)=0 .
$$


In the light of the five functionals fixed point theorem, they established the criteria for the existence of at least three solutions for the dynamic equation (1.1) either with conditions (1.4) or with conditions (1.5).

More recently, Yaslan $[27,28]$ investigated the dynamic equation:

$$
u^{\Delta \nabla}(t)+h(t) f(t, u(t))=0, \quad t \in\left[t_{1}, t_{3}\right]_{\mathbb{T}} \subset \mathbb{T},
$$

which satisfies either the boundary value conditions

$$
\alpha u\left(t_{1}\right)-\beta_{0} u^{\Delta}\left(t_{1}\right)=u^{\Delta}\left(t_{2}\right), \quad u^{\Delta}\left(t_{3}\right)=0,
$$

or the conditions

$$
u^{\Delta}\left(t_{1}\right)=0, \quad \alpha u\left(t_{3}\right)+\beta u^{\Delta}\left(t_{3}\right)=u^{\Delta}\left(t_{2}\right)
$$

Here, $0 \leqslant t_{1}<t_{2}<t_{3}, \alpha>0, \beta_{0} \geqslant 0$, and $\beta>1$. Indeed, Yaslan analytically established the conditions for the existence of at least two or three positive solutions in these boundary value problems by virtue of the Avery-Henderson fixed point theorem and the Leggett-Williams fixed point theorem [36]. It is worthwhile to mention that these theoretical results are novel even for some special cases on time scales, such as the conventional difference equations with fixed time stepsize and the ordinary differential equations.

Motivated by the aforementioned results and techniques in coping with those boundary value problems on time scales, we thus turn to investigate the possible existence of multiple positive solutions for the following one-dimensional $p$-Laplacian dynamic equation:

$$
\left(\phi_{p}\left(u^{\Delta}(t)\right)\right)^{\nabla}+h(t) f(t, u(t))=0, \quad t \in(0, T]_{\mathbb{T}}
$$

with the $p$-Laplacian and $m$-point boundary value conditions:

$$
\phi_{p}\left(u^{\Delta}(0)\right)=\sum_{i=1}^{m-2} a_{i} \phi_{p}\left(u^{\Delta}\left(\xi_{i}\right)\right), \quad u(T)+\beta B_{0}\left(u^{\Delta}(T)\right)=\sum_{i=1}^{m-2} B\left(u^{\Delta}\left(\xi_{i}\right)\right) .
$$

In the following discussion, we implement three hypotheses as follows.

$\left(\mathrm{H}_{1}\right)$ One has $a_{i} \geqslant 0$ for $i=1, \ldots, m-2,0<\xi_{1}<\xi_{2}<\cdots<\xi_{m-2}<T$, and $d_{0}=1-\sum_{i=1}^{m-2} a_{i}>0$.

$\left(\mathrm{H}_{2}\right)$ One has that $h:[0, \sigma(T)]_{\mathbb{T}} \mapsto[0, \infty)$ is left dense continuous (ld-continuous), and there exists a $t_{0} \in[0, T]_{\mathbb{T}}$ such that $h\left(t_{0}\right) \neq 0$. Also $f:[0, \sigma(T)]_{\mathbb{T}} \times[0, \infty) \mapsto[0, \infty)$ is continuous.

$\left(\mathrm{H}_{3}\right)$ Both $B_{0}$ and $B$ are continuously odd functions defined on $\mathbb{R}$. There exist two positive numbers $\underline{b}$ and $\bar{b}$ such that, for any $v>0$,

$$
\underline{b} v \leqslant B_{0}(v), \quad B(v) \leqslant \bar{b} v
$$


and that

$$
\beta \underline{b}-(m-2) \bar{b}-\mu(T) \geqslant 0
$$

It is clear that, together with conditions (1.10) and the above hypotheses $\left(\mathrm{H}_{1}\right)-\left(\mathrm{H}_{3}\right)$, the dynamic equation (1.9) not only covers the corresponding boundary value problems in the literature, but even nontrivially generalizes these problems to a much wider class of boundary value problems on time scales. Also it is valuable to mention that condition (1.12) in hypothesis $\left(\mathrm{H}_{3}\right)$ is necessarily relevant to the graininess operator $\mu: \tau \rightarrow[0,+\infty)$ around the time instant $T$. Such kind of condition has not been required in the literature, to the best of authors' knowledge. Thus, this paper analytically establishes some new and time-scale-dependent criteria for the existence of at least double or triple positive solutions in the boundary value problems (1.9) and (1.10) by virtue of the Avery-Henderson fixed point theorem and the five functionals fixed point theorem. Indeed, these obtained criteria significantly extend the results existing in [26-28].

The remainder of the paper is organized as follows. Section 2 preliminarily provides some lemmas which are crucial to the following discussion. Section 3 analytically establishes the criteria for the existence of at least two positive solutions in the boundary value problems (1.9) and (1.10) with the aid of the Avery-Henderson fixed point theorem. Section 4 gives some sufficient conditions for the existence of at least three positive solutions by means of the five functionals fixed point theorem. More importantly, Section 5 provides a representative and nontrivial example to illustrate a possible application of the obtained analytical results on dynamic equations on time scales. Finally, the paper is closed with some concluding remarks.

\section{Preliminaries}

In this section, we intend to provide several lemmas which are crucial to the proof of the main results in this paper. However, for concision, we omit the introduction of those elementary notations and definitions, which can be found in $[11,12,33]$ and references therein.

The following lemmas are based on the following linear boundary value problems:

$$
\begin{gathered}
\left(\phi_{p}\left(u^{\Delta}(t)\right)\right)^{\nabla}+g(t)=0, \quad t \in(0, T]_{\mathbb{T}}, \\
\phi_{p}\left(u^{\Delta}(0)\right)=\sum_{i=1}^{m-2} a_{i} \phi_{p}\left(u^{\Delta}\left(\xi_{i}\right)\right), \quad u(T)+\beta B_{0}\left(u^{\Delta}(T)\right)=\sum_{i=1}^{m-2} B\left(u^{\Delta}\left(\xi_{i}\right)\right) .
\end{gathered}
$$


Lemma 2.1. Assume that $d_{0}=1-\sum_{i=1}^{m-2} a_{i} \neq 0$. Then, for $g \in C_{\mathrm{ld}}[0, T]_{\mathbb{T}}$, the linear boundary value problems (2.1) have a unique solution satisfying

$$
\begin{aligned}
u(t)= & \int_{t}^{T} \phi_{q}\left(\int_{0}^{s} g(\tau) \nabla \tau+\frac{1}{d_{0}} \sum_{i=1}^{m-2} a_{i} \int_{0}^{\xi_{i}} g(\tau) \nabla \tau\right) \Delta s \\
& +\beta B_{0}\left(\phi_{q}\left(\int_{0}^{T} g(\tau) \nabla \tau+\frac{1}{d_{0}} \sum_{i=1}^{m-2} a_{i} \int_{0}^{\xi_{i}} g(\tau) \nabla \tau\right)\right) \\
& -\sum_{i=1}^{m-2} B\left(\phi_{q}\left(\int_{0}^{\xi_{i}} g(\tau) \nabla \tau+\frac{1}{d_{0}} \sum_{i=1}^{m-2} a_{i} \int_{0}^{\xi_{i}} g(\tau) \nabla \tau\right)\right),
\end{aligned}
$$

for all $t \in[0, \sigma(T)]_{\mathbb{T}}$.

Proof. According to the formula $\left(\int_{a}^{t} f(t, s) \Delta s\right)^{\Delta}=f(\sigma(t), t)+\int_{a}^{t} f(t, s) \Delta s$ introduced in [12], we have

$$
u^{\Delta}(t)=\phi_{q}\left(-\int_{0}^{t} g(\tau) \nabla \tau-\frac{1}{d_{0}} \sum_{i=1}^{m-2} a_{i} \int_{0}^{\xi_{i}} g(\tau) \nabla \tau\right) .
$$

Thus, we obtain that

$$
\phi_{p}\left(u^{\Delta}(t)\right)=-\int_{0}^{t} g(\tau) \nabla \tau-\frac{1}{d_{0}} \sum_{i=1}^{m-2} a_{i} \int_{0}^{\xi_{i}} g(\tau) \nabla \tau,
$$

and that

$$
\left(\phi_{p}\left(u^{\Delta}(t)\right)\right)^{\nabla}=-g(t)
$$

To this end, it is not hard to check that $u(t)$ satisfies (2.2), which implies that $u(t)$ is a solution of the problems (2.1).

Furthermore, in order to verify the uniqueness, we suppose that both $u_{1}(t)$ and $u_{2}(t)$ are the solutions of the problems (2.1). Then, we have

$$
\begin{gathered}
\left(\phi_{p}\left(u_{1}^{\Delta}(t)\right)\right)^{\nabla}-\left(\phi_{p}\left(u_{2}^{\Delta}(t)\right)\right)^{\nabla}=0, \quad t \in(0, T]_{\mathbb{T}}, \\
\phi_{p}\left(u_{1}^{\Delta}(0)\right)-\phi_{p}\left(u_{2}^{\Delta}(0)\right)=\sum_{i=1}^{m-2} a_{i}\left[\phi_{p}\left(u_{1}^{\Delta}\left(\xi_{i}\right)\right)-\phi_{p}\left(u_{2}^{\Delta}\left(\xi_{i}\right)\right)\right], \\
u_{1}(T)-u_{2}(T)+\beta B_{0}\left(u_{1}^{\Delta}(T)\right)-\beta B_{0}\left(u_{2}^{\Delta}(T)\right)=\sum_{i=1}^{m-2}\left[B\left(u_{1}^{\Delta}\left(\xi_{i}\right)\right)-B\left(u_{2}^{\Delta}\left(\xi_{i}\right)\right)\right] .
\end{gathered}
$$


According to Theorem A.5 in [37], (2.6) further yields

$$
\phi_{p}\left(u_{1}^{\Delta}(t)\right)-\phi_{p}\left(u_{2}^{\Delta}(t)\right)=\bar{c}, \quad t \in[0, T]_{\mathbb{T}} .
$$

Hence, from (2.7) and (2.9), the assumption $d_{0}=1-\sum_{i=1}^{m-2} a_{i} \neq 0$, and the definition of the $p$-Laplacian operator, it follows that

$$
u_{1}^{\Delta}(t)-u_{2}^{\Delta}(t) \equiv 0, \quad t \in[0, T]_{\mathbb{T}} .
$$

This equation, together with (2.8), further implies

$$
u_{1}(t) \equiv u_{2}(t), \quad t \in[0, \sigma(T)]_{\mathbb{T}},
$$

which consequently leads to the completion of the proof, that is, $u(t)$ specified in $(2.2)$ is the unique solution of the problems (2.1).

Lemma 2.2. Assume that $d_{0}=1-\sum_{i=1}^{m-2} a_{i}>0$ and that $\beta \underline{b}-(m-2) \bar{b}-\mu(T) \geqslant 0$. If $g \in$ $C_{\mathrm{ld}}\left([0, \sigma(T)]_{\mathbb{T}},[0, \infty)\right)$, then the unique solution of the problems (2.1) satisfies

$$
u(t) \geqslant 0, \quad t \in[0, \sigma(T)]_{\mathbb{T}}
$$

Proof. By (2.2) specified in Lemma 2.1, we get

$$
u^{\Delta}(t)=-\phi_{q}\left(\int_{0}^{t} g(\tau) \nabla \tau+\frac{1}{d_{0}} \sum_{i=1}^{m-2} a_{i} \int_{0}^{\xi_{i}} g(\tau) \nabla \tau\right) \leqslant 0, \quad t \in[0, T]_{\mathbb{T}} .
$$

Thus, $u(t)$ is nonincreasing in the interval $[0, \sigma(T)]_{\mathbb{T}}$. In addition, notice that

$$
\begin{aligned}
u(\sigma(T))= & \int_{\sigma(T)}^{T} \phi_{q}\left(\int_{0}^{s} g(\tau) \nabla \tau+\frac{1}{d_{0}} \sum_{i=1}^{m-2} a_{i} \int_{0}^{\xi_{i}} g(\tau) \nabla \tau\right) \Delta s \\
& +\beta B_{0}\left(\phi_{q}\left(\int_{0}^{T} g(\tau) \nabla \tau+\frac{1}{d_{0}} \sum_{i=1}^{m-2} a_{i} \int_{0}^{\xi_{i}} g(\tau) \nabla \tau\right)\right) \\
& -\sum_{i=1}^{m-2} B\left(\phi_{q}\left(\int_{0}^{\xi_{i}} g(\tau) \nabla \tau+\frac{1}{d_{0}} \sum_{i=1}^{m-2} a_{i} \int_{0}^{\xi_{i}} g(\tau) \nabla \tau\right)\right)
\end{aligned}
$$




$$
\begin{aligned}
= & -\mu(T) \phi_{q}\left(\int_{0}^{T} g(\tau) \nabla \tau+\frac{1}{d_{0}} \sum_{i=1}^{m-2} a_{i} \int_{0}^{\xi_{i}} g(\tau) \nabla \tau\right) \\
& +\beta B_{0}\left(\phi_{q}\left(\int_{0}^{T} g(\tau) \nabla \tau+\frac{1}{d_{0}} \sum_{i=1}^{m-2} a_{i} \int_{0}^{\xi_{i}} g(\tau) \nabla \tau\right)\right) \\
& -\sum_{i=1}^{m-2} B\left(\phi_{q}\left(\int_{0}^{\xi_{i}} g(\tau) \nabla \tau+\frac{1}{d_{0}} \sum_{i=1}^{m-2} a_{i} \int_{0}^{\xi_{i}} g(\tau) \nabla \tau\right)\right) \\
\geqslant & {[\beta \underline{b}-(m-2) \bar{b}-\mu(T)]\left[\phi_{q}\left(\int_{0}^{T} g(\tau) \nabla \tau+\frac{1}{d_{0}} \sum_{i=1}^{m-2} a_{i} \int_{0}^{\xi_{i}} g(\tau) \nabla \tau\right)\right] . }
\end{aligned}
$$

The last term in the above estimation is no less than zero because of the assumptions. Thus, from the monotonicity of $u(t)$, we get

$$
u(t) \geqslant u(\sigma(T)) \geqslant 0, \quad t \in[0, \sigma(T)]_{\mathbb{T}}
$$

which completes the proof.

Now, denote that $\mathcal{\varepsilon}=C_{\mathrm{ld}}[0, \sigma(T)]_{\mathbb{T}}$ and that $\|u\|=\sup _{t \in[0, \sigma(T)]_{\mathbb{T}}}|u(t)|$, where $u \in \mathcal{\varepsilon}$. Thus, it is easy to verify that $\mathcal{\varepsilon}$ endowed with $\|\cdot\|$ becomes a Banach space. Furthermore, define a cone, denoted by $p$, through,

$$
\begin{aligned}
D=\{u \in \mathcal{E} \mid & u(t) \geqslant 0 \text { for } t \in[0, \sigma(T)]_{\mathbb{T}} \\
& \left.u^{\Delta}(t) \leqslant 0 \text { for } t \in[0, T]_{\mathbb{T}}, u^{\Delta \nabla}(t) \leqslant 0 \text { for } t \in(0, \sigma(T))_{\mathbb{T}}\right\} .
\end{aligned}
$$

Also, for a given positive real number $r$, define a function set $p_{r}$ by

$$
p_{r}=\{u \in P \mid\|u\|<r\} .
$$

Naturally, we denote that $\bar{D}_{r}=\{u \in P \mid\|u\| \leqslant r\}$ and that $\partial p_{r}=\{u \in D \mid\|u\|=r\}$. With these settings, we have the following properties.

Lemma 2.3. If $u \in D$, then (i) $u(t) \geqslant((T-t) / T)\|u\|$ for any $t \in[0, T]_{\mathbb{T}}$ (ii) $(T-s) u(t) \geqslant$ $(T-t) u(s)$ for any pair of $s, t \in[0, T]_{\mathbb{T}}$ with $t \geqslant s$. 
The proof of this lemma, which could be found in [26, 28], is directly from the specific construction of the set $P$. Next, let us construct a map $\mathfrak{A}: D \rightarrow \varepsilon$ through

$$
\begin{aligned}
{[\mathfrak{A} u](t)=} & \int_{t}^{T} \phi_{q}\left(\int_{0}^{s} h(\tau) f(\tau, u(\tau)) \nabla \tau+\frac{1}{d_{0}} \sum_{i=1}^{m-2} a_{i} \int_{0}^{\xi_{i}} h(\tau) f(t, u(\tau)) \nabla \tau\right) \Delta s \\
& +\beta B_{0}\left(\phi_{q}\left(\int_{0}^{T} h(\tau) f(\tau, u(\tau)) \nabla \tau+\frac{1}{d_{0}} \sum_{i=1}^{m-2} a_{i} \int_{0}^{\xi_{i}} h(\tau) f(\tau, u(\tau)) \nabla \tau\right)\right) \\
& -\sum_{i=1}^{m-2} B\left(\phi_{q}\left(\int_{0}^{\xi_{i}} h(\tau) f(\tau, u(\tau)) \nabla \tau+\frac{1}{d_{0}} \sum_{i=1}^{m-2} a_{i} \int_{0}^{\xi_{i}} h(\tau) f(\tau, u(\tau)) \nabla \tau\right)\right),
\end{aligned}
$$

for any $u \in P$. Then, through a standard argument [33], it is not hard to validate the following properties on this map.

Lemma 2.4. Assume that the hypotheses $\left(H_{1}\right)-\left(H_{3}\right)$ are all fulfilled. Then, $\mathfrak{A}(\mathcal{D}) \subset \mathcal{D}$, and $\mathfrak{A}: \bar{p}_{r} \rightarrow$ $D$ is completely continuous.

\section{At Least Two Positive Solutions in Boundary Value Problems}

In this section, we aim to adopt the well-known Avery-Henderson fixed point theorem to prove the existence of at least two positive solutions in the boundary value problems (1.9) and (1.10). For the sake of self-containment, we first state the Avery-Henderson fixed point theorem as follows.

Theorem 3.1 (see [34]). Let $p$ be a cone in a real Banach space $\varepsilon$. For each $d>0$, set $p(\psi, d)=$ $\{x \in D \mid \psi(x)<d\}$. Let $\alpha$ and rbe increasing, nonnegative continuous functionals on $D$, and let $\theta$ be a nonnegative continuous functional on $D$ with $\theta(0)=0$ such that, for some $c>0$ and $H>0$,

$$
\gamma(x) \leqslant \theta(x) \leqslant \alpha(x), \quad\|x\| \leqslant H \gamma(x),
$$

for all $x \in \overline{p(\gamma, c)}$. Suppose that there exist a completely continuous operator $\mathfrak{A}: \overline{p(\gamma, c)} \rightarrow D$ and three positive numbers $0<a<b<c$ such that

$$
\theta(\lambda x) \leqslant \lambda \theta(x), \quad 0 \leqslant \lambda \leqslant 1, \quad x \in \partial p(\theta, b),
$$

and (i) $\gamma(\mathfrak{A} x)>c$ for all $x \in \partial p(\gamma, c)$, (ii) $\theta(\mathfrak{A} x)<b$ for all $x \in \partial P(\theta, b)$, and (iii) $D(\alpha, a) \neq \emptyset$ and $\alpha(\mathfrak{A} x)>$ a for all $x \in \partial \mathcal{P}(\alpha, a)$. Then, the operator $\mathfrak{A}$ has at least two fixed points, denoted by $x_{1}$ and $x_{2}$, belonging to $\overline{D(\gamma, c)}$ and satisfying $a<\alpha\left(x_{1}\right)$ with $\theta\left(x_{1}\right)<b$ and $b<\theta\left(x_{2}\right)$ with $\gamma\left(x_{2}\right)<c$. 
Now, set $t^{\star}=\min \{t \in \mathbb{T} \mid T / 2 \leqslant t \leqslant T\}$ and select $t_{\star} \in \mathbb{T}$ satisfying $0<t_{\star}<t^{\star}$. Denote, respectively, that

$$
\begin{gathered}
M=\frac{T-t^{\star}}{T} \int_{0}^{t^{\star}} \phi_{q}\left(\int_{0}^{s} h(\tau) \nabla \tau\right) \Delta s, \\
N=(T+\beta \bar{b}) \cdot \phi_{q}\left(\frac{1}{d_{0}} \int_{0}^{T} h(\tau) \nabla \tau\right), \\
L=\frac{T-t^{\star}}{T} \int_{t_{\star}}^{T} \phi_{q}\left(\int_{t_{\star}}^{s} h(\tau) \nabla \tau\right) \Delta s, \\
L_{0}=\left[T-t_{\star}+\beta \bar{b}-(m-2) \underline{b}\right] \cdot \phi_{q}\left(\frac{1}{d_{0}} \int_{0}^{T} h(\tau) \nabla \tau\right) .
\end{gathered}
$$

Hence, we are in a position to obtain the following results.

Theorem 3.2. Assume that the hypotheses $\left(H_{1}\right)-\left(H_{3}\right)$ all hold and that there exist positive real numbers $a, b, c$ such that

$$
0<a<b<c, \quad a<\frac{L}{N} b<\frac{L\left(T-t^{\star}\right)}{T L} c .
$$

In addition, assume that $f$ satisfies the following conditions:

$\left(C_{1}\right) f(t, u)>\phi_{p}(c / M)$ for $t \in\left[0, t^{\star}\right]_{\mathbb{T}}$ and $u \in\left[c,\left(T /\left(T-t^{\star}\right)\right) c\right]$;

$\left(C_{2}\right) f(t, u)<\phi_{p}(b / N)$ for $t \in[0, T]_{\mathbb{T}}$ and $u \in\left[0,\left(T /\left(T-t^{\star}\right)\right) b\right]$;

$\left(C_{3}\right) f(t, u)>\phi_{p}(a / L)$ for $t \in\left[t_{\star}, T\right]_{\mathbb{T}}$ and $u \in[0, a]$.

Then, the boundary value problems (1.9) and (1.10) have at least two positive solutions $u_{1}$ and $u_{2}$ such that

$$
\begin{aligned}
& a<\max _{t \in\left[t_{*}, T\right]_{\mathbb{T}}} u_{1}(t) \text { with } \max _{t \in\left[t^{*}, T\right]_{\mathbb{T}}} u_{1}(t)<b, \\
& b<\max _{t \in\left[t^{*}, T\right]_{\mathbb{T}}} u_{1}(t) \text { with } \min _{t \in\left[t_{*}, t^{*}\right]_{\mathbb{T}}} u_{2}(t)<c .
\end{aligned}
$$

Proof. Construct the cone $P$ and the operator $\mathfrak{A}$ as specified in (2.16) and (2.18), respectively. In addition, define the increasing, nonnegative, and continuous functionals $\gamma, \theta$, and $\alpha$ on $p$, respectively, by

$$
\begin{gathered}
\gamma(u)=\min _{t \in\left[t_{\star}, t^{\star}\right]_{\mathbb{T}}} u(t)=u\left(t^{\star}\right), \quad \theta(u)=\max _{t \in\left[t^{\star}, T\right]_{\mathbb{T}}} u(t)=u\left(t^{\star}\right), \\
\alpha(u)=\max _{t \in\left[t_{\star}, T\right]_{\mathbb{T}}} u(t)=u\left(t_{\star}\right) .
\end{gathered}
$$

Evidently, $\gamma(u)=\theta(u) \leqslant \alpha(u)$ for each $u \in p$. 
In addition, for each $u \in D$, Lemma 2.3 manifests that $\gamma(u)=u\left(t^{\star}\right) \geqslant\left(\left(T-t^{\star}\right) / T\right)\|u\|$. Thus, we have

$$
\|u\| \leqslant \frac{T}{T-t^{\star}} \gamma(u)
$$

for each $u \in D$. Also, notice that $\theta(\lambda u)=\lambda \theta(u)$ for $\lambda \in[0,1]$ and $u \in \partial P(\theta, b)$. Furthermore, from Lemma 2.4, it follows that the operator $\mathfrak{A}: \overline{p(\gamma, c)} \rightarrow D$ is completely continuous.

In what follows, we are to verify that all the conditions of Theorem 3.1 are satisfied with respect to the operator $\mathfrak{A}$.

Let $u \in \partial p(\gamma, c)$. Then, $\gamma(u)=\min _{t \in\left[t_{*}, t^{\star}\right]_{\mathbb{T}}} u(t)=u\left(t^{\star}\right)=c$. This implies that $u(t) \geqslant c$ for $t \in\left[0, t^{\star}\right]_{\mathbb{T}}$, which, combined with (3.7), yields

$$
c \leqslant u(t) \leqslant \frac{T}{T-t^{\star}} c
$$

for $t \in\left[0, t^{\star}\right]_{\mathbb{T}}$. Because of assumption $\left(C_{1}\right), f(t, u(t))>\phi_{p}(c / M)$ for $t \in\left[0, t^{\star}\right]_{\mathbb{T}}$. According to the specific form in (2.18), Lemma 2.3 , and the property $\mathfrak{A} u \in \mathcal{D}$, we obtain that

$$
\begin{aligned}
& \gamma(\mathfrak{A} u) \\
& =[\mathfrak{A} u]\left(t^{\star}\right) \geqslant \frac{T-t^{\star}}{T}\|\mathfrak{A} u\|=\frac{T-t^{\star}}{T}[\mathfrak{A} u](0) \\
& =\frac{T-t^{\star}}{T}\left[\int_{0}^{T} \phi_{q}\left(\int_{0}^{s} h(\tau) f(\tau, u(\tau)) \nabla \tau+\frac{1}{d_{0}} \sum_{i=1}^{m-2} a_{i} \int_{0}^{\xi_{i}} h(\tau) f(\tau, u(\tau)) \nabla \tau\right) \Delta s\right. \\
& +\beta B_{0}\left(\phi_{q}\left(\int_{0}^{T} h(\tau) f(\tau, u(\tau)) \nabla \tau+\frac{1}{d_{0}} \sum_{i=1}^{m-2} a_{i} \int_{0}^{\xi_{i}} h(\tau) f(\tau, u(\tau)) \nabla \tau\right)\right) \\
& \left.-\sum_{i=1}^{m-2} B\left(\phi_{q}\left(\int_{0}^{\xi_{i}} f(t, u(\tau)) \nabla \tau+\frac{1}{d_{0}} \sum_{i=1}^{m-2} a_{i} \int_{0}^{\xi_{i}} h(\tau) f(\tau, u(\tau)) \nabla \tau\right)\right)\right] \\
& \geqslant \frac{T-t^{\star}}{T}\left[\int_{0}^{T} \phi_{q}\left(\int_{0}^{s} h(\tau) f(\tau, u(\tau)) \nabla \tau+\frac{1}{d_{0}} \sum_{i=1}^{m-2} a_{i} \int_{0}^{\xi_{i}} h(\tau) f(\tau, u(\tau)) \nabla \tau\right) \Delta s\right. \\
& \left.+(\beta \underline{b}-(m-2) \bar{b}) \phi_{q}\left(\int_{0}^{T} h(\tau) f(\tau, u(\tau)) \nabla \tau+\frac{1}{d_{0}} \sum_{i=1}^{m-2} a_{i} \int_{0}^{\xi_{i}} h(\tau) f(\tau, u(\tau)) \nabla \tau\right)\right] \\
& \geqslant \frac{T-t^{\star}}{T} \int_{0}^{T} \phi_{q}\left(\int_{0}^{s} h(\tau) f(\tau, u(\tau)) \nabla \tau\right) \Delta s \\
& \geqslant \frac{T-t^{\star}}{T} \int_{0}^{t^{\star}} \phi_{q}\left(\int_{0}^{s} h(\tau) f(\tau, u(\tau)) \nabla \tau\right) \Delta s
\end{aligned}
$$




$$
\begin{aligned}
& \geqslant \frac{T-t^{\star}}{T} \int_{0}^{t^{\star}} \phi_{q}\left(\int_{0}^{s} h(\tau) f(\tau, u(\tau)) \nabla \tau\right) \Delta s \\
& >\frac{T-t^{\star}}{T} \cdot \frac{c}{M} \cdot \int_{0}^{t^{\star}} \phi_{q}\left(\int_{0}^{s} h(\tau) \nabla \tau\right) \Delta s=c .
\end{aligned}
$$

Thus, condition (i) in Theorem 3.1 is satisfied.

Next, consider $u \in \partial P(\theta, b)$. In such a case, we have $\gamma(u)=\theta(u)=\max _{t \in\left[t^{*}, T\right]_{\mathbb{T}}} u(t)=$ $u\left(t^{\star}\right)=b$, which implies that $0 \leqslant u(t) \leqslant b$ for $t \in\left[t^{\star}, T\right]_{\mathbb{T}}$. Analogously, it follows from (3.7) that, for all $u \in D$,

$$
\|u\| \leqslant \frac{T}{T-t^{\star}} \gamma(u)=\frac{T}{T-t^{\star}} b .
$$

Therefore, we obtain $0 \leqslant u(t) \leqslant\left(T /\left(T-t^{\star}\right)\right) b$ for $t \in[0, T]_{\mathbb{T}}$. This, combined with assumption $\left(C_{2}\right)$, gives $f(t, u(t))<\phi_{p}(b / N)$ for all $t \in[0, T]_{\mathbb{T}}$. Thus, we have

$$
\begin{aligned}
\theta(\mathfrak{A} u)= & \max _{t \in\left[t^{\star}, T\right]_{\mathbb{T}}}[\mathfrak{A} u](t)=[\mathfrak{A} u]\left(t^{\star}\right) \leqslant[\mathfrak{A} u](0) \\
= & \int_{0}^{T} \phi_{q}\left(\int_{0}^{s} h(\tau) f(\tau, u(\tau)) \nabla \tau+\frac{1}{d_{0}} \sum_{i=1}^{m-2} a_{i} \int_{0}^{\xi_{i}} h(\tau) f(\tau, u(\tau)) \nabla \tau\right) \Delta s \\
& +\beta B_{0}\left(\phi_{q}\left(\int_{0}^{T} h(\tau) f(\tau, u(\tau)) \nabla \tau+\frac{1}{d_{0}} \sum_{i=1}^{m-2} a_{i} \int_{0}^{\xi_{i}} h(\tau) f(\tau, u(\tau)) \nabla \tau\right)\right) \\
& -\sum_{i=1}^{m-2} B\left(\phi_{q}\left(\int_{0}^{\xi_{i}} f(t, u(\tau)) \nabla \tau+\frac{1}{d_{0}} \sum_{i=1}^{m-2} a_{i} \int_{0}^{\xi_{i}} h(\tau) f(\tau, u(\tau)) \nabla \tau\right)\right) \\
\leqslant & \int_{0}^{T} \phi_{q}\left(\frac{1}{d_{0}} \int_{0}^{T} h(\tau) f(\tau, u(\tau)) \nabla \tau\right) \Delta s+\beta \bar{b} \phi_{q}\left(\frac{1}{d_{0}} \int_{0}^{T} h(\tau) f(\tau, u(\tau)) \nabla \tau\right) \\
< & \frac{b}{N}\left[\int_{0}^{T} \phi_{q}\left(\frac{1}{d_{0}} \int_{0}^{T} h(\tau) \nabla \tau\right) \Delta s+\beta \bar{b} \phi_{q}\left(\frac{1}{d_{0}} \int_{0}^{T} h(\tau) \nabla \tau\right)\right] \\
= & \frac{b(T+\beta \bar{b})}{N} \cdot \phi_{q}\left(\frac{1}{d_{0}} \int_{0}^{T} h(\tau) \nabla \tau\right)=b,
\end{aligned}
$$

which consequently implies the validity of condition (ii) in Theorem 3.1. 
Finally, notice that the constant functions $(1 / 2) a \in D(\alpha, a)$, so that $D(\alpha, a) \neq \emptyset$. Let $u \in \partial P(\alpha, a)$. Then, we get $\alpha(u)=\max _{t \in\left[t_{\star}, T\right]_{T}} u(t)=u\left(t_{\star}\right)=a$. This with assumption $\left(C_{3}\right)$ implies that $0 \leqslant u(t) \leqslant a$ and $f(t, u)>\phi_{p}(a / L)$ for all $t \in\left[t_{\star}, T\right]_{\mathbb{T}}$. Similarly, we have

$$
\begin{aligned}
\alpha(\mathfrak{A} u)=[\mathfrak{A} u]\left(t_{\star}\right) \geqslant \frac{T-t_{\star}}{T}[\mathfrak{A} u](0) & \\
= & \frac{T-t_{\star}}{T}\left[\int_{0}^{T} \phi_{q}\left(\int_{0}^{s} h(\tau) f(\tau, u(\tau)) \nabla \tau+\frac{1}{d_{0}} \sum_{i=1}^{m-2} a_{i} \int_{0}^{\xi_{i}} h(\tau) f(\tau, u(\tau)) \nabla \tau\right) \Delta s\right. \\
& +\beta \phi_{q}\left(\int_{0}^{T} h(\tau) f(\tau, u(\tau)) \nabla \tau+\frac{1}{d_{0}} \sum_{i=1}^{m-2} a_{i} \int_{0}^{\xi_{i}} h(\tau) f(\tau, u(\tau)) \nabla \tau\right) \\
\geqslant & \frac{T-t_{\star}}{T} \int_{t_{\star}}^{T} \phi_{q}\left(\int_{0}^{s} h(\tau) f(\tau, u(\tau)) \nabla \tau\right) \Delta s \\
\geqslant & \frac{T-t_{\star}}{T} \int_{t_{\star}}^{T} \phi_{q}\left(\int_{t_{\star}}^{s} h(\tau) f(\tau, u(\tau)) \nabla \tau\right) \Delta s \\
> & \left.\frac{a}{L} \cdot \frac{T-t_{\star}}{T} \cdot \int_{t_{\star}}^{\xi_{i}} \phi_{q}\left(\phi_{q}(t, u(\tau)) \nabla \tau+\frac{1}{d_{0}} \sum_{i=1}^{m-2} a_{i} \int_{0}^{\xi_{i}} h(\tau) f(\tau, u(\tau)) \nabla \tau\right)\right]
\end{aligned}
$$

Indeed, the validity of condition (iii) in Theorem 3.1 is verified.

According to Theorem 3.1, we consequently approach the conclusion that the boundary value problems (1.9) and (1.10) possess at least two positive solutions, denoted by $u_{1}$ and $u_{2}$, satisfying $a<\alpha\left(u_{1}\right)$ with $\theta\left(u_{1}\right)<b$ and $b<\theta\left(u_{2}\right)$ with $\gamma\left(u_{2}\right)<c$, respectively.

\section{At Least Three Positive Solutions in Boundary Value Problems}

In this section, we are to prove the existence of at least three positive solutions in the boundary value problems (1.9) and (1.10) by using the five functionals fixed point theorem which is attributed to Avery [35].

Let $\gamma, \beta, \theta$ be nonnegative continuous convex functionals on $p . \alpha$ and $\psi$ are supposed to be nonnegative continuous concave functionals on $p$. Thus, for nonnegative real numbers $h, a, b, c$, and $d$, define five convex sets, respectively, by

$$
\begin{gathered}
D(\gamma, c)=\{x \in P \mid \gamma(x)<c\}, \\
D(\gamma, \alpha, a, c)=\{x \in D \mid a \leqslant \alpha(x), \gamma(x) \leqslant c\}, \\
\mathcal{Q}(\gamma, \beta, d, c)=\{x \in P \mid \beta(x) \leqslant d, \gamma(x) \leqslant c\}, \\
D(\gamma, \theta, \alpha, a, b, c)=\{x \in D \mid a \leqslant \alpha(x), \theta(x) \leqslant b, \gamma(x) \leqslant c\}, \\
Q(\gamma, \beta, \psi, h, d, c)=\{x \in D \mid h \leqslant \psi(x), \beta(x) \leqslant d, \gamma(x) \leqslant c\} .
\end{gathered}
$$


Theorem 4.1 (see [35]). Let $p$ be a cone in a real Banach space $\varepsilon$. Suppose that $\alpha$ and $\psi$ are nonnegative continuous concave functionals on $p$, and that $\gamma, \beta$, and $\theta$ are nonnegative continuous convex functionals on $P$ such that, for some positive numbers $c$ and $M$,

$$
\alpha(x) \leqslant \beta(x), \quad\|x\| \leqslant M \gamma(x),
$$

for all $x \in \overline{p(\gamma, c)}$. In addition, suppose that $\mathfrak{A}: \overline{p(\gamma, c)} \mapsto \overline{p(\gamma, c)}$ is a completely continuous operator and that there exist nonnegative real numbers $h, d, a, b$ with $0<d<a$ such that

(i) $\{x \in P(\gamma, \theta, \alpha, a, b, c) \mid \alpha(x)>a\} \neq \emptyset$ and $\alpha(\mathfrak{A} x)>$ a for $x \in P(\gamma, \theta, \alpha, a, b, c)$;

(ii) $\{x \in \mathcal{Q}(\gamma, \beta, \psi, h, d, c) \mid \beta(x)<d\} \neq \emptyset$ and $\beta(\mathfrak{A} x)<d$ for $x \in \mathcal{Q}(\gamma, \beta, \psi, h, d, c)$;

(iii) $\alpha(\mathfrak{A} x)>$ a for $x \in P(\gamma, \alpha, a, c)$ with $\theta(\mathfrak{A} x)>b$;

(iv) $\beta(\mathfrak{A} x)<d$ for $x \in \mathcal{Q}(\gamma, \beta, d, c)$ with $\psi(\mathfrak{A} x)<h$.

Then the operator $\mathfrak{A}$ admits at least three fixed points $x_{1}, x_{2}, x_{3} \in \overline{D(\gamma, c)}$ satisfying $\beta\left(x_{1}\right)<d$, $a<\alpha\left(x_{2}\right)$, and $d<\beta\left(x_{3}\right)$ with $\alpha\left(x_{3}\right)<a$, respectively.

With this theorem, we are now in a position to establish the following result on the existence of at least three solutions in the boundary value problems (1.9) and (1.10).

Theorem 4.2. Suppose that the hypotheses $\left(H_{1}\right)-\left(H_{3}\right)$ are all fulfilled. Assume that there exist positive real numbers $a, b, c$ such that

$$
0<a<b<c, \quad a<\frac{T-t_{\star}}{T} b<\frac{\left(T-t_{\star}\right)\left(T-t^{\star}\right)}{T^{2}} c, \quad N b<M c .
$$

Also assume that $f$ satisfies the following conditions:

$\left(C_{1}\right) f(t, u)<\phi_{p}(c / N)$ for $t \in[0, T]_{\mathbb{T}}$ and $u \in\left[0,\left(T /\left(T-t^{\star}\right)\right) c\right]$;

$\left(C_{2}\right) f(t, u)>\phi_{p}(b / M)$ for $t \in\left[0, t^{\star}\right]_{\mathbb{T}}$ and $u \in\left[b,\left(T^{2} /\left(T-t^{\star}\right)^{2}\right) b\right]$;

$\left(C_{3}\right) f(t, u)<\phi_{p}\left(a / L_{0}\right)$ for $t \in[0, T]_{\mathbb{T}}$ and $u \in\left[0,\left(T /\left(T-t_{\star}\right)\right) a\right]$.

Then, the boundary value problems (1.9) and (1.10) admit at least three solutions $u_{1}(t), u_{2}(t)$, and $u_{3}(t)$, defined on $[0, \sigma(T)]_{\mathbb{T}}$, satisfying, respectively,

$$
\begin{aligned}
& \max _{t \in\left[t_{*}, T\right]_{\mathbb{T}}} u_{1}(t)<a, \quad b<\min _{t \in\left[0, t_{\star}\right]_{\mathbb{T}}} u_{2}(t), \\
& a<\max _{t \in\left[t_{*}, T\right]_{\mathbb{T}}} u_{3}(t) \quad \text { with } \min _{t \in\left[0, t_{*}\right]_{\mathbb{T}}} u_{3}(t)<b .
\end{aligned}
$$


Proof. Let the cone $P$ be as constructed in (2.16) and the operator $\mathfrak{A}$ as defined in (2.18). Define, respectively, the nonnegative continuous concave functionals on the $D$ as follows:

$$
\begin{gathered}
r(u)=\theta(u)=\max _{t \in\left[t^{\star}, T\right]_{\mathbb{T}}} u(t)=u\left(t^{\star}\right), \\
\alpha(u)=\min _{t \in\left[0, t_{\star}\right]_{\mathbb{T}}} u(t)=u\left(t_{\star}\right), \\
\beta(u)=\max _{t \in\left[t_{\star}, T\right]_{\mathbb{T}}} u(t)=u\left(t_{\star}\right), \\
\psi(u)=\min _{t \in\left[0, t^{\star}\right]_{\mathbb{T}}} u(t)=u\left(t^{\star}\right) .
\end{gathered}
$$

Thus, we get $\alpha(u)=\beta(u)$ for $u \in D$. Moreover, from Lemma 2.3, it follows that

$$
\|u\| \leqslant \frac{T}{T-t^{\star}} \gamma(u)
$$

for $u \in P$. Next, we intend to verify that all the conditions in Theorem 4.1 hold with respect to the operator $\mathfrak{A}$.

To this end, arbitrarily pick up a function $u \in \overline{p(\gamma, c)}$. Then, $\gamma(u)=\max _{t \in\left[t^{\star}, T\right]_{\mathbb{T}}} u(t)=$ $u\left(t^{\star}\right) \leqslant c$, which, combined with (4.6), implies that $0 \leqslant u(t) \leqslant\left(T /\left(T-t^{\star}\right)\right) c$ for $t \in[0, T]_{\mathbb{T}}$ and $u \in D$. Thus, we have $f(t, u(t))<\phi_{p}(c / N)$ for $t \in[0, T]_{\mathbb{T}}$, owing to assumption $\left(C_{1}\right)$. Moreover, since $\mathfrak{A} u \in D$, we have

$$
\begin{aligned}
\|\gamma(\mathfrak{A} u)\|= & {[\mathfrak{A} u]\left(t^{\star}\right) \leqslant[\mathfrak{A} u](0) } \\
= & \int_{0}^{T} \phi_{q}\left(\int_{0}^{s} h(\tau) f(\tau, u(\tau)) \nabla \tau+\frac{1}{d_{0}} \sum_{i=1}^{m-2} a_{i} \int_{0}^{\xi_{i}} h(\tau) f(\tau, u(\tau)) \nabla \tau\right) \Delta s \\
& +\beta B_{0}\left(\phi_{q}\left(\int_{0}^{T} h(\tau) f(\tau, u(\tau)) \nabla \tau+\frac{1}{d_{0}} \sum_{i=1}^{m-2} a_{i} \int_{0}^{\xi_{i}} h(\tau) f(\tau, u(\tau)) \nabla \tau\right)\right) \\
& -\sum_{i=1}^{m-2} B\left(\phi_{q}\left(\int_{0}^{\xi_{i}} f(t, u(\tau)) \nabla \tau+\frac{1}{d_{0}} \sum_{i=1}^{m-2} a_{i} \int_{0}^{\xi_{i}} h(\tau) f(\tau, u(\tau)) \nabla \tau\right)\right) \\
\leqslant & \int_{0}^{T} \phi_{q}\left(\frac{1}{d_{0}} \int_{0}^{T} h(\tau) f(\tau, u(\tau)) \nabla \tau\right) \Delta s+\beta \bar{b} \phi_{q}\left(\frac{1}{d_{0}} \int_{0}^{T} h(\tau) f(\tau, u(\tau)) \nabla \tau\right) \\
< & \frac{c}{N}\left[\int_{0}^{T} \phi_{q}\left(\frac{1}{d_{0}} \int_{0}^{T} h(\tau) \nabla \tau\right) \Delta s+\beta \bar{b} \phi_{q}\left(\frac{1}{d_{0}} \int_{0}^{T} h(\tau) \nabla \tau\right)\right] \\
= & \frac{c(T+\beta \bar{b})}{N} \cdot \phi_{q}\left(\frac{1}{d_{0}} \int_{0}^{T} h(\tau) \nabla \tau\right)=c .
\end{aligned}
$$

This, with Lemma 2.4, clearly manifests that the operator $\mathfrak{A}: \overline{p(\gamma, c)} \mapsto \overline{p(\gamma, c)}$ is completely continuous. 
Moreover, the set

$$
\left\{u \in p\left(r, \theta, \alpha, b, \frac{T}{T-t^{\star}} b, c\right) \mid \alpha(u)>b\right\}
$$

is not empty, because the constant function $u(t) \equiv\left(\left(2 T-t^{\star}\right) / 2\left(T-t^{\star}\right)\right) b$ belongs to the set $\left\{u \in P\left(\gamma, \theta, \alpha, b,\left(T /\left(T-t^{\star}\right)\right) b, c\right) \mid \alpha(u)>b\right\}$. Analogously, the set

$$
\left\{u \in Q\left(\gamma, \beta, \psi, \frac{T-t^{\star}}{T} a, a, c\right) \mid \beta(u)<a\right\}
$$

is nonempty, since $u(t) \equiv\left(\left(T+t^{\star}\right) / 2 T\right) a \in\left\{u \in \mathcal{Q}\left(\gamma, \beta, \psi,\left(\left(T-t^{\star}\right) / T\right) a, a, c\right) \mid \beta(u)<a\right\}$. For particular $u \in D\left(\gamma, \theta, \alpha, b,\left(T /\left(T-t^{\star}\right)\right) b, c\right)$, a utilization of (4.6) produces

$$
b \leqslant \min _{t \in\left[0, t^{\star}\right]_{\mathbb{T}}} u(t)=u\left(t^{\star}\right) \leqslant u(t) \leqslant \frac{T}{T-t^{\star}} \gamma(u)=\frac{T}{\left(T-t^{\star}\right)} \theta(u) \leqslant \frac{T^{2}}{\left(T-t^{\star}\right)^{2}} b,
$$

for $t \in\left[0, t^{\star}\right]_{\mathbb{T}}$. According to assumption $\left(C_{2}\right)$, we thus obtain

$$
f(t, u(t))>\phi_{p}\left(\frac{b}{M}\right)
$$

for all $t \in\left[0, t^{\star}\right]_{\mathbb{T}}$. Hence, it follows from (4.11) and Lemma 2.3 that

$$
\begin{aligned}
\alpha(\mathfrak{A} u)=[\mathfrak{A} u]\left(t_{\star}\right) \geqslant \frac{T-t_{\star}}{T}[\mathfrak{A}] u(0) & \frac{T-t_{\star}}{T}\left[\int_{0}^{T} \phi_{q}\left(\int_{0}^{s} h(\tau) f(\tau, u(\tau)) \nabla \tau+\frac{1}{d_{0}} \sum_{i=1}^{m-2} a_{i} \int_{0}^{\xi_{i}} h(\tau) f(\tau, u(\tau)) \nabla \tau\right) \Delta s\right. \\
& +\beta B_{0}\left(\phi_{q}\left(\int_{0}^{T} h(\tau) f(\tau, u(\tau)) \nabla \tau+\frac{1}{d_{0}} \sum_{i=1}^{m-2} a_{i} \int_{0}^{\xi_{i}} h(\tau) f(\tau, u(\tau)) \nabla \tau\right)\right) \\
\geqslant & \frac{T-t_{\star}}{T} \int_{0}^{t^{\star}} \phi_{q}\left(\int_{0}^{s-2} h(\tau) f(\tau, u(\tau)) \nabla \tau\right) \Delta s \\
> & \left.\left.\frac{b}{M} \cdot \frac{T-t_{\star}}{T} \cdot \int_{0}^{t^{\star}} \phi_{q}\left(\int_{0}^{s} h(t, u(\tau)) \nabla \tau+\frac{1}{d_{0}} \sum_{i=1}^{m-2} a_{i} \int_{0}^{\xi_{i}} h(\tau) f(\tau, u(\tau)) \nabla \tau\right)\right)\right]
\end{aligned}
$$

This definitely verifies the validity of condition (i) in Theorem 4.1. 
Next, let us consider $u \in \mathcal{Q}\left(\gamma, \beta, \psi,\left(\left(T-t^{\star}\right) / T\right) a, a, c\right)$. In this case, we get

$$
0 \leqslant u(t) \leqslant \frac{T}{T-t_{\star}} a,
$$

for $t \in[0, T]_{\mathbb{T}}$. Thus, an adoption of the assumption $\left(C_{3}\right)$ yields $f(t, u(t))<\phi_{p}\left(a / L_{0}\right)$. Furthermore, we have

$$
\begin{aligned}
\beta(\mathfrak{A} u)= & {[\mathfrak{A} u]\left(t_{\star}\right) } \\
= & \int_{t_{\star}}^{T} \phi_{q}\left(\int_{0}^{s} h(\tau) f(\tau, u(\tau)) \nabla \tau+\frac{1}{d_{0}} \sum_{i=1}^{m-2} a_{i} \int_{0}^{\xi_{i}} h(\tau) f(t, u(\tau)) \nabla \tau\right) \Delta s \\
& +\beta B_{0}\left(\phi_{q}\left(\int_{0}^{T} h(\tau) f(\tau, u(\tau)) \nabla \tau+\frac{1}{d_{0}} \sum_{i=1}^{m-2} a_{i} \int_{0}^{\xi_{i}} h(\tau) f(\tau, u(\tau)) \nabla \tau\right)\right) \\
& -\sum_{i=1}^{m-2} B\left(\phi_{q}\left(\int_{0}^{\xi_{i}} h(\tau) f(\tau, u(\tau)) \nabla \tau+\frac{1}{d_{0}} \sum_{i=1}^{m-2} a_{i} \int_{0}^{\xi_{i}} h(\tau) f(\tau, u(\tau)) \nabla \tau\right)\right) \\
\leq & \left(T-t_{\star}+\beta \bar{b}-(m-2) \underline{b}\right) \phi_{q}\left(\frac{1}{d_{0}} \int_{0}^{T} h(\tau) f(\tau, u(\tau)) \nabla \tau\right) \\
< & \frac{a}{L_{0}}\left(T-t_{\star}+\beta \bar{b}-(m-2) \underline{b}\right) \cdot \phi_{q}\left(\frac{1}{d_{0}} \int_{0}^{T} h(\tau) \nabla \tau\right)=a .
\end{aligned}
$$

Accordingly, the validity of condition (ii) in Theorem 4.1 is verified.

Aside from conditions (i) and (ii), we are finally to verify the validity of conditions (iii) and (iv). For this purpose, on the one hand, consider $u \in p(\gamma, \alpha, b, c)$ with $\theta(\mathfrak{A} u)>$ $\left(T /\left(T-t^{\star}\right)\right) b$. Thus, we have

$$
\alpha(\mathfrak{A} u)=[\mathfrak{A} u]\left(t_{\star}\right) \geqslant[\mathfrak{A} u]\left(t^{\star}\right)=\theta(\mathfrak{A} u)>\frac{T}{T-t^{\star}} b>b .
$$

On the other hand, consider $u \in \mathcal{Q}(\gamma, \beta, a, c)$ with $\psi(\mathfrak{A} u)<\left(\left(T-t^{\star}\right) / T\right) a$. In such a case, we obtain that

$$
\beta(\mathfrak{A} u)=[\mathfrak{A} u]\left(t_{\star}\right) \leqslant \frac{T-t_{\star}}{T-t^{\star}}[\mathfrak{A} u]\left(t^{\star}\right)=\frac{T-t_{\star}}{T-t^{\star}} \psi(\mathfrak{A} u)<\frac{T-t_{\star}}{T} a<a .
$$

Therefore, both conditions (iii) and (iv) in Theorem 4.1 are satisfied. Consequently, by virtue of Theorem 4.1, the boundary value problems (1.9) and (1.10) have at least three positive solutions circumscribed on $[0, \sigma(T)]_{\mathbb{T}}$ satisfying $\max _{t \in\left[t_{*}, T\right]_{\mathbb{T}}} u_{1}(t)<a, b<\min _{t \in\left[0, t_{*}\right]_{\mathbb{T}}} u_{2}(t)$, and $a<\max _{t \in\left[t_{*}, T\right]_{\mathbb{T}}} u_{3}(t)$ with $\min _{t \in\left[0, t_{\star}\right]_{\mathbb{T}}} u_{3}(t)<b$. 


\section{A Specific Example}

In this section, we provide a representative and nontrivial example to clearly illustrate the feasibility of the time-scale-dependent results of dynamic equations with boundary value conditions that are obtained in the preceding section.

Construct a nontrivial time-scale set as

$$
\mathbb{T}=\left\{1-\left(\frac{1}{2}\right)^{\mathbb{N}^{0}}\right\} \cup[1,2] \cup\left\{T^{\star}\right\} .
$$

Take all the parameters in problems (1.9) and (1.10) as follows: $T=2,2<T^{\star} \leqslant 3, p=3 / 2$, $q=3, m=4, a_{1}=a_{2}=1 / 4, \underline{b}=1 / 2, \bar{b}=1, \beta=6, \xi_{1}=1 / 2, \xi_{2}=1, t^{\star}=1$, and $t_{\star}=1 / 2$, so that $d_{0}=1 / 2$. For simplicity but without loss of generality, set $h(t) \equiv 1$. Also notice that there exist countable right-scattered points $t_{i}=1-(1 / 2)^{i},(i=0,1,2, \ldots)$. Then, it is easy to validate the condition

$$
\beta \underline{b}-(m-2) \bar{b}-\mu(T) \geqslant 0
$$

which is dependent on the time scale property around the time instant $T$. Furthermore, implementing the integral formula [38]:

$$
\int_{a}^{b} f(s) \Delta s=\int_{a}^{b} f(s) \mathrm{d} s+\sum_{t_{i} \in[a, b)_{\mathbb{T}}} \int_{t_{i}}^{\sigma\left(t_{i}\right)}\left[f\left(t_{i}\right)-f(s)\right] \mathrm{d} s,
$$

we concretely obtain that

$$
\begin{aligned}
M & =\frac{T-t^{\star}}{T} \int_{0}^{t^{\star}} \phi_{q}\left(\int_{0}^{s} h(\tau) \nabla \tau\right) \Delta s \\
& =\int_{0}^{1} s^{2} \Delta s=\int_{0}^{1} s^{2} \mathrm{~d} s+\sum_{i=1}^{\infty} \int_{t_{i}}^{\sigma\left(t_{i}\right)}\left(t_{i}^{2}-s^{2}\right) \mathrm{d} s=\frac{5}{21} \\
N & =(T+\beta \bar{b}) \cdot \phi_{q}\left(\frac{1}{d_{0}} \int_{0}^{T} h(\tau) \nabla \tau\right)=128 \\
L_{0} & =\left[T-t_{\star}+\beta \bar{b}-(m-2) \underline{b}\right] \cdot \phi_{q}\left(\frac{1}{d_{0}} \int_{0}^{T} h(\tau) \nabla \tau\right)=104 .
\end{aligned}
$$

Particularly, take the function in dynamic equation as

$$
f(t, u)=\frac{23 u^{2}}{16+t+u+u^{2}}, \quad t \in[0,2]_{\mathbb{T}}, u \geqslant 0 .
$$


This kind of function is omnipresent in the mathematical modeling of biological or chemical processes. Then it allows us to properly set the other parameters as $a=1 / 416, b=105$, and $c=1078 N$. It is evident that these parameters satisfy

$$
0<a<\frac{T-t_{\star}}{T} b<\frac{\left(T-t_{\star}\right)\left(T-t^{\star}\right)}{T^{2}} c, \quad N b<M c .
$$

Now, we can verify the validity of conditions $\left(C_{1}\right)-\left(C_{3}\right)$ in Theorem 4.2 . Indeed, direct computations yield:

$$
f(t, u)<23=\left(\frac{c}{N}\right)^{1 / 2}=\phi_{p}\left(\frac{C}{N}\right)
$$

as $t \in[0, T]_{\mathbb{T}}$ and $u \in[0,2 c]$,

$$
f(t, u) \geqslant \frac{23 b^{2}}{16+t_{\star}+b+b^{2}}>21=\phi_{p}\left(\frac{b}{M}\right)
$$

as $t \in\left[0, t_{\star}\right]_{\mathbb{T}}$ and $u \in[b, 4 b]$, and

$$
f(t, u) \leqslant \frac{23 u^{2}}{16} \leqslant 23 a^{2}<2 a=\frac{1}{112}=\phi_{p}\left(\frac{a}{L_{0}}\right)
$$

as $t \in[0, T]_{\mathbb{T}}$ and $u \in[0,4 a]$. Hence, conditions $\left(C_{1}\right)-\left(C_{3}\right)$ in Theorem 4.2 are satisfied for the above specified functions and parameters. Therefore, in the light of Theorem 4.2, we conclude that the dynamic equation on the specified time scales

$$
\left[\left(u^{\Delta}\right)^{1 / 2}\right]^{\nabla}+\frac{23 u^{2}}{16+t+u+u^{2}}=0, \quad t \in(0,2)_{\mathbb{T}}
$$

with the boundary value conditions

$$
\begin{gathered}
{\left[u^{\Delta}(0)\right]^{1 / 2}=\frac{1}{4}\left[u^{\Delta}\left(\frac{1}{2}\right)\right]^{1 / 2}+\frac{1}{4}\left[u^{\Delta}(1)\right]^{1 / 2},} \\
u(2)+6 u^{\Delta}(2)=\frac{1}{2} u^{\Delta}\left(\frac{1}{2}\right)+\frac{1}{2} u^{\Delta}(1)
\end{gathered}
$$

has at least three positive solutions defined on $\left[0, T^{\star}\right]_{\mathbb{T}}$ satisfying $\max _{t \in\left[t_{\star}, T\right]_{\mathbb{T}}} u_{1}(t)<a, b<$ $\min _{t \in\left[0, t_{\star}\right]_{\mathbb{T}}} u_{2}(t)$, and $a<\max _{t \in\left[t_{*}, T\right]_{\mathbb{T}}} u_{3}(t)$ with $\min _{t \in\left[0, t_{\star}\right]_{\mathbb{T}}} u_{3}(t)<b$.

\section{Concluding Remarks}

In this paper, some novel and time-scale-dependent sufficient conditions are established for the existence of multiple positive solutions in a specific kind of boundary value problems 
on time scales. This kind of boundary value problems not only includes the problems discussed in the literature but also is adapted to more general cases. The well-known AveryHenderson fixed point theorem and the five functionals fixed point theorem are adopted in the arguments.

It is valuable to mention that the writing form of the ending point of the interval on time scales should be accurately specified in dealing with different kind of boundary value conditions. Any inaccurate expression may lead to a problematic or incomplete discussion. Also it is noted that some other fixed point theorems and degree theories may be adapted to dealing with various boundary value problems on time scales. In addition, future directions for further generalization of the boundary value problem on time scales may include the generalization of the $p$-Laplacian operator to increasing homeomorphism and homeomorphism, which has been investigated in [39] for the nonlinear boundary value of ordinary differential equations; the allowance of the function $f$ to change sign, which has been discussed in [31] and needs more detailed and rigorous investigations.

\section{Acknowledgments}

This paper was supported by the NNSF of China (Grants nos. 10501008 and 60874121) and by the Rising-Star Program Foundation of Shanghai, China (Grant no. 07QA14002). The authors are grateful to the referee and editors for their very helpful suggestions and comments.

\section{References}

[1] B. Aulbach and S. Hilger, "Linear dynamic processes with inhomogeneous time scale," in Nonlinear Dynamics and Quantum Dynamical Systems (Gaussig, 1990), vol. 59 of Mathematics Research, pp. 9-20, Akademie, Berlin, Germany, 1990.

[2] S. Hilger, "Analysis on measure chains-a unified approach to continuous and discrete calculus," Results in Mathematics, vol. 18, no. 1-2, pp. 18-56, 1990.

[3] R. P. Agarwal and M. Bohner, "Basic calculus on time scales and some of its applications," Results in Mathematics, vol. 35, no. 1-2, pp. 3-22, 1999.

[4] R. P. Agarwal, M. Bohner, and P. Rehak, "Half-linear dynamic equations," in Nonlinear Analysis and Applications: To V. Lakshmikantham on His 80th Birthday. Vol. 1, 2, pp. 1-57, Kluwer Academic Publishers, Dordrecht, The Netherlands, 2003.

[5] V. Lakshmikantham, S. Sivasundaram, and B. Kaymakcalan, Dynamic Systems on Measure Chains, vol. 370 of Mathematics and Its Applications, Kluwer Academic Publishers, Dordrecht, The Netherlands, 1996.

[6] J. Hoffacker and C. C. Tisdell, "Stability and instability for dynamic equations on time scales," Computers E Mathematics with Applications, vol. 49, no. 9-10, pp. 1327-1334, 2005.

[7] W. Zhong, W. Lin, and J. Ruan, "The generalized invariance principle for dynamic equations on time scales," Applied Mathematics and Computation, vol. 184, no. 2, pp. 557-565, 2007.

[8] H. Wu and Z. Zhou, "Stability for first order delay dynamic equations on time scales," Computers $\mathcal{E}$ Mathematics with Applications, vol. 53, no. 12, pp. 1820-1831, 2007.

[9] V. Otero-Espinar and D. R. Vivero, "Uniqueness and existence results for initial value problems on time scales through a reciprocal problem and applications," Computers \& Mathematics with Applications, vol. 58, no. 4, pp. 700-710, 2009.

[10] D. R. Anderson and P. J. Y. Wong, "Positive solutions for second-order semipositone problems on time scales," Computers \& Mathematics with Applications, vol. 58, no. 2, pp. 281-291, 2009.

[11] M. Bohner and A. Peterson, Eds., Advances in Dynamic Equations on Time Scales, Birkhäuser, Boston, Mass, USA, 2003.

[12] M. Bohner and A. Peterson, Dynamic Equations on Time Scales. An Introduction with Applications, Birkhäuser, Boston, Mass, USA, 2001.

[13] F. M. Atici, D. C. Biles, and A. Lebedinsky, "An application of time scales to economics," Mathematical and Computer Modelling, vol. 43, no. 7-8, pp. 718-726, 2006. 
[14] E. Akin, "Boundary value problems for a differential equation on a measure chain," Panamerican Mathematical Journal, vol. 10, no. 3, pp. 17-30, 2000.

[15] R. P. Agarwal and D. O’Regan, "Triple solutions to boundary value problems on time scales," Applied Mathematics Letters, vol. 13, no. 4, pp. 7-11, 2000.

[16] R. P. Agarwal and D. O'Regan, "Nonlinear boundary value problems on time scales," Nonlinear Analysis: Theory, Methods \& Applications, vol. 44, no. 4, pp. 527-535, 2001.

[17] D. R. Anderson, "Solutions to second-order three-point problems on time scales," Journal of Difference Equations and Applications, vol. 8, no. 8, pp. 673-688, 2002.

[18] D. R. Anderson, "Nonlinear triple-point problems on time scales," Electronic Journal of Differential Equations, no. 47, 12 pages, 2004.

[19] E. R. Kaufmann, "Positive solutions of a three-point boundary-value problem on a time scale," Electronic Journal of Differential Equations, no. 82, 11 pages, 2003.

[20] J. J. DaCunha, J. M. Davis, and P. K. Singh, "Existence results for singular three point boundary value problems on time scales," Journal of Mathematical Analysis and Applications, vol. 295, no. 2, pp. 378-391, 2004.

[21] Z. He, "Existence of two solutions of $m$-point boundary value problem for second order dynamic equations on time scales," Journal of Mathematical Analysis and Applications, vol. 296, no. 1, pp. 97-109, 2004.

[22] H.-R. Sun and W.-T. Li, "Multiple positive solutions for $p$-Laplacian $m$-point boundary value problems on time scales," Applied Mathematics and Computation, vol. 182, no. 1, pp. 478-491, 2006.

[23] H.-R. Sun and W.-T. Li, "Existence theory for positive solutions to one-dimensional $p$-Laplacian boundary value problems on time scales," Journal of Differential Equations, vol. 240, no. 2, pp. 217$248,2007$.

[24] Y.-H. Su and W.-T. Li, “Triple positive solutions of $m$-point BVPs for $p$-Laplacian dynamic equations on time scales," Nonlinear Analysis: Theory, Methods E Applications, vol. 69, no. 11, pp. 3811-3820, 2008.

[25] Z. He, "Double positive solutions of three-point boundary value problems for $p$-Laplacian dynamic equations on time scales," Journal of Computational and Applied Mathematics, vol. 182, no. 2, pp. 304-315, 2005.

[26] Z. He and L. Li, "Multiple positive solutions for the one-dimensional $p$-Laplacian dynamic equations on time scales," Mathematical and Computer Modelling, vol. 45, no. 1-2, pp. 68-79, 2007.

[27] İ. Yaslan, "Multiple positive solutions for nonlinear three-point boundary value problems on time scales," Computers \& Mathematics with Applications, vol. 55, no. 8, pp. 1861-1869, 2008.

[28] İ. Yaslan, "Existence of positive solutions for nonlinear three-point problems on time scales," Journal of Computational and Applied Mathematics, vol. 206, no. 2, pp. 888-897, 2007.

[29] D. R. Anderson and I. Y. Karaca, "Higher-order three-point boundary value problem on time scales," Computers \& Mathematics with Applications, vol. 56, no. 9, pp. 2429-2443, 2008.

[30] D. R. Anderson, R. Avery, and J. Henderson, "Existence of solutions for a one dimensional $p$-Laplacian on time-scales," Journal of Difference Equations and Applications, vol. 10, no. 10, pp. 889-896, 2004.

[31] Y. Sang, H. Su, and F. Xu, "Positive solutions of nonlinear $m$-point BVP for an increasing homeomorphism and homomorphism with sign changing nonlinearity on time scales," Computers E Mathematics with Applications, vol. 58, no. 2, pp. 216-226, 2009.

[32] H.-R. Sun, "Triple positive solutions for $p$-Laplacian $m$-point boundary value problem on time scales," Computers \& Mathematics with Applications, vol. 58, no. 9, pp. 1736-1741, 2009.

[33] W. Zhong and W. Lin, "Existence of positive solutions in generalized boundary value problem for $p$-Laplacian dynamic equations on time scales," Advances in Difference Equations, vol. 2009, Article ID 848191, 19 pages, 2009.

[34] R. I. Avery and J. Henderson, "Two positive fixed points of nonlinear operators on ordered Banach spaces," Communications on Applied Nonlinear Analysis, vol. 8, no. 1, pp. 27-36, 2001.

[35] R. I. Avery, "A generalization of the Leggett-Williams fixed point theorem," Mathematical Sciences Research Hot-Line, vol. 3, no. 7, pp. 9-14, 1999.

[36] R. W. Leggett and L. R. Williams, "Multiple positive fixed points of nonlinear operators on ordered Banach spaces," Indiana University Mathematics Journal, vol. 28, no. 4, pp. 673-688, 1979.

[37] G. Sh. Guseinov, "Integration on time scales," Journal of Mathematical Analysis and Applications, vol. 285, no. 1, pp. 107-127, 2003.

[38] A. Cabada and D. R. Vivero, "Expression of the Lebesgue $\Delta$-integral on time scales as a usual Lebesgue integral: application to the calculus of $\Delta$-antiderivatives," Mathematical and Computer Modelling, vol. 43, no. 1-2, pp. 194-207, 2006.

[39] C. Bereanu and J. Mawhin, "Existence and multiplicity results for some nonlinear problems with singular $\phi$-Laplacian," Journal of Differential Equations, vol. 243, no. 2, pp. 536-557, 2007. 